\title{
An alternative proof for the linear utility representation theorem ${ }^{\star}$
}

\author{
Walter Trockel ${ }^{1}$ \\ ${ }^{1}$ Institute for Mathematical Economics, Bielefeld University, Postbox 8640, W-4800 Bielefeld 1, Federa! \\ Republic of Germany
}

Received: February 20, 1991; revised version June 15, 1991

Summary. The paper presents an alternative short proof for the linear utility representation theorem. In particular a generalization of the theorem of Blackwell and Girshick (1954) and a special case of the theorem of Herstein and Milnor (1953) are proved by exploiting the topological group structure of finite-dimensional Euclidean vector space.

\section{Introduction}

Linear Utility Representation results play a prominent role in economic theory. Apart from their significance for decision theory under uncertainty, they found applications in social choice theory and in demand theory.

In particular the version due to Blackwell and Girshick (1954) has been used to derive several versions of a utilitarianism theorm by d'Aspremont and Gevers (1977), Maskin (1978), Gevers (1979), Roberts (1980). (cf. also d'Aspremont (1985)).

Looking at formulations of axioms from which linear utility functions may be derived one easily recognizes the presence of invariance postulates. The theorems of Blackwell and Girshick (1954) and of Herstein and Milnor (1953) are respectively, based on invariance postulates for the preference relation and the indifference relation.

These facts suggest to base a proof of this theorem on a systematic use of group theory. As continuity has to play its part one is forced to use topological groups. It turns out that standard arguments lead to a short and simple proof. In the proof of our version of the Herstein and Milnor result (our proposition) we have to pay for the abridgement by restricting to $\mathbb{R}^{\prime}$ rather than working in general mixture sets. Regarding Blackwell and Girshick's result our corollary does not bring about very much of abbreviation. It provides however quite a different approach yielding a linear utility without imposing the assumption of monotonicity of preferences used by Blackwell and Girshick.

\footnotetext{
* I thank two careful referees for their helpful remarks.
} 
We shall collect the necessary notation and definitions in Sect. 2.

Section 3 contains the results and proofs. The paper ends with concluding remarks in Sect. 4.

\section{The framework}

Let $R$ be a preference relation on $\mathbb{R}^{\ell}, \ell \in \mathbb{N}$, i.e. a complete transitive binary relation on $\mathbb{R}^{\ell}$. For given $R$ we denote by $I$ and $P$, respectively, the induced indifference and strict preference relation. Formally:

$$
\begin{aligned}
\forall x, y \in \mathbb{R}^{\ell}: & x I y \Leftrightarrow x R y \text { and } y R x \\
& x P y \Leftrightarrow x R y \text { and not } y R x .
\end{aligned}
$$

We shall use the following notation:

$$
\begin{array}{ll}
R(x):=\left\{y \in \mathbb{R}^{\ell} \mid x R y\right\} & R^{-1}(x):=\left\{y \in \mathbb{R}^{\ell} \mid y R x\right\} \\
P(x):=\left\{y \in \mathbb{R}^{\ell} \mid x P y\right\} & P^{-1}(x):=\left\{y \in \mathbb{R}^{\ell} \mid y P x\right\} \\
I(x):=\left\{y \in \mathbb{R}^{\ell} \mid x I y\right\}=: I^{-1}(x) .
\end{array}
$$

For convenience we shall denote the $R$-indifference set $I(x)$ of $x$ by $I_{x}$ for any $x \in \mathbb{R}^{\ell}$.

Definition 1. The preference relation $R$ on $\mathbb{R}^{\ell}$ is upper (resp. lower) semi-continuous at $x \in \mathbb{R}^{\ell}$ iff $R^{-1}(x)$ (resp. $R(x)$ ) is closed. $R$ is upper (resp. lower) semi-continuous if it is so at every $x \in \mathbb{R}^{\ell}$. The preference relation $R$ is continuous (at $x \in \mathbb{R}^{\ell}$ ) iff it is upper and lower semi-continuous (at $x \in \mathbb{R}^{\ell}$ ).

Recall that $R$ is upper resp. lower semi-continuous resp. continuous iff it allows an upper resp. lower semi-continuous resp. continuous utility representation.

Definition 2. A binary relation $T$ on $\mathbb{R}^{\ell}$ is called (translation-) invariant iff

$$
\forall x, y, z \in \mathbb{R}^{\ell}: x T y \Leftrightarrow x+z T y+z .
$$

Definition 3. A preference relation $R$ on $\mathbb{R}^{\ell}$ is trivial iff $R=I$.

\section{Results}

Lemma. Assume that $R$ is non-trivial and continuous and $I$ is translation-invariant. Then there is a hyperplane $H$ of $\mathbb{R}^{\ell}$ through 0 such that

(i) $\forall x \in \mathbb{R}^{t}: I_{x}=x+H$

(ii) The two open half spaces into which $H$ separates $\mathbb{R}^{\ell}$ coincide with $P(0)$ and $P^{-1}(0)$.

Proof. As $I_{0}=R^{-1}(0) \cap R(0)$ it is closed by continuity of $R$. For $x, y \in I_{0}$ invariance yields $x-y,-y \in I_{0}$. So $I_{0}$ is a closed subgroup of $\mathbb{R}^{\ell}$. Again by invariance we get $n x+m y \in I_{0}$ for any $n, m \in \mathbb{Z}$ and thus for any $n, m \in \mathbb{Q}$. Hence $I_{0}$ is a linear subspace of $\mathbb{R}^{\ell}$.

Since $R$ is non-trivial there exists $z \in P^{-1}(0)$. We will show that this implies $-z \in P(0)$. First, $-z \in I_{0}$ is impossible since by invariance it would imply $z \in I_{0}$, a 
contradiction. Assume $-z \in P^{-1}(0)$. Then $z I-z$ is impossible because by invariance it would imply $2 z \in I_{0}$, hence $z \in I_{0}$, again a contradiction. So assume without loss of generality $z P-z P 0$. Then $[0, z]=([0, z] \cap P(-z)) \cup\left([0, z] \cap P^{-1}(-z)\right)$ is impossible, as both sets in the union are non-empty, relatively open in $[0, z]$ and disjoint. Therefore there exists $z^{\prime} \in[0, z] \cap I(-z)$. As $0 \in\left[-z, z^{\prime}\right]$ this implies $-z \in I_{0}$, a contradiction.

So the open sets $P(0)$ and $P^{-1}(0)$ are disjoint and non-empty with $P^{-1}(0) \cup P(0)=$ $\mathbb{R}^{\complement} \backslash I_{0}$. Hence $\mathbb{R}^{\ell} \backslash I_{0}$ cannot be connected which implies $\operatorname{dim} I_{0} \geqq \ell-1$. Nontriviality then yields $\operatorname{dim} I_{0}=l-1$. Since for $H:=I_{0}$ and any $x \in \mathbb{R}^{\ell}$ the set $I_{x}=x+H$ is a hyperplane (i) is established.

Next let $z P 0$ and $p$ be a linear functional on $\mathbb{R}^{\ell}$ with kernel $H$ and $p \cdot z>0$. Let $H_{+}:=\left\{y \in \mathbb{R}^{\ell} \mid p \cdot y>0\right\}$ and $H_{-}:=-H_{+}$. Since $H_{+}, H_{-}, P(0), P^{-1}(0)$ are nonempty and open and $H_{+}, H_{-}$are connected and since $z P 0$, the equality $H_{+}=$ $\left(H_{+} \cap P(0)\right) \cup\left(H_{+} \cap P^{-1}(0)\right)$ implies that $H_{+} \cap P(0)=\varnothing$. This implies $H_{+} \subset P^{-1}(0)$ and $P(0) \subset H_{-}$. Since both $\left\{P^{-1}(0), P(0)\right\}$ and $\left\{H_{+}, H_{-}\right\}$cover $\mathbb{R}^{\complement} \backslash H$ equality of $P^{-1}(0)$ and $H_{+}$is equivalent to equality of $P(0)$ and $H$. Assume $P^{-1}(0) \cap H_{-} \neq \varnothing$ and $z^{\prime} \in P^{-1}(0) \cap H_{-}$. As was shown above this implies $-z^{\prime} \in P(0) \cap H_{+}$, a contradiction. Hence, $P^{-1}(0)=H_{+}$and thus, $P(0)=H$. Q.E.D.

Next we shall use this lemma to prove for the mixture set $\mathbb{R}^{\ell}$ the linear utility representation theorem of Herstein and Milnor (1953). It should be noted however that the longer original proof holds true for any mixture set while the present proof exploits the specific framework of a finite-dimensional space.

Proposition. Let $R$ be continuous with translation-invariant $I$. Then $R$ is representable by a linear utility function.

Proof. A trivial $R$ is represented by the linear map $x \mapsto 0$. Now let $R$ be non-trivial. By the lemma $I_{0}$ is a linear subspace, in particular the kernel of the linear functional p. Moreover,

$$
H_{+}=\left\{y \in \mathbb{R}^{\ell} \mid p \cdot y>0\right\}=P^{-1}(0) .
$$

To show that $p$ represents $R$ means to establish

$$
\forall x, y \in \mathbb{R}^{\ell}: p \cdot x \geqq p \cdot y \Leftrightarrow x R y .
$$

As $x I y$ if and only if $p \cdot x=p \cdot y$ it suffices to show that

$$
\forall x, y \in \mathbb{R}^{\ell}: p \cdot x>p \cdot y \Rightarrow x P y .
$$

If $p \cdot x>0>p \cdot y$ the lemma yields $x P 0 P y$.

Now let $p \cdot x>p \cdot y>0$. Since $I_{y}$ is a hyperplane separating $I_{x}$ from

$$
y+H_{-} \text {and as }[0, y] \subset y+H_{-} \text {we get }[0, y] \cap I_{x}=\varnothing .
$$

Hence $[0, y]=([0, y] \cap P(x)) \cup\left([0, y] \cap P^{-1}(x)\right)$. As $0 \in P(x)$ and $[0, y]$ is closed and connected $[0, y] \cap P^{-1}(x)=\varnothing$, hence $x P y$.

Finally, let $0>p \cdot x>p \cdot y$.

Since $[0, x]=([0, x] \cap P(y)) \cup\left([0, x] \cap P^{-1}(y)\right)$ and $0 \in P^{-1}(y)$ we have $[0, x] \cap$ $P(y)=\varnothing$, thus $x P y$. Q.E.D. 
The next result generalizes the linear utility representation theorem due to Blackwell and Girshick (1954, Theorem 4.3.1 and the associated problem).

Corollary. Let $R$ be translation-invariant and semi-continuous at some point $x \in \mathbb{R}^{\ell}$. Then $R$ is representable by a linear utility function.

Proof. W.l.o.g. assume $R$ to be non-trivial. Translation-invariance implies $R(x)=$ $x+R(0), R^{-1}(x)=x+R^{-1}(0)$ for any $x \in \mathbb{R}^{\ell}$ and $R^{-1}(0)=-R(0)$. Therefore semicontinuity of $R$ at some $x$ implies continuity of $R$. Now the assumptions of the proposition are fulfilled. Q.E.D.

It is immediate that any preference relation represented by a linear utility function must have the properties stated in the assumptions of the two theorems. Therefore these respective sets of assumptions are sufficient and necessary.

\section{Concluding remarks}

The proofs for the two theorems are based on the structure of $\mathbb{R}^{\ell}$ as a finite dimensional topological group and are in fact quite different from the original ones.

The continuity assumption in the framework of general mixture sets of Herstein and Milnor is formulated in a different way. However, if the mixture set is a finite-dimensional Euclidean space the continuity used there (Axiom 2) coincides with the usual one. While full continuity is used in our proposition (and in Herstein and Milnor (1953)) translation invariance is required only for the associated indifference relation.

Our corollary does not depend on monotonicity. The proof of Blackwell and Girshick (1954), however, crucially depends on that assumption. As the two versions of the linear utility representation result show there is a trade-off between invariance and continuity. Full continuity allows to derive translation-invariance of the preference relation from that of the associated indifference relation. One the other hand, translation invariance of the preference relation allows to derive full continuity from semi-continuity at some point.

One might be tempted to try to derive linear utility representability from a combination of the weaker continuity and the weaker invariance assumption. This is not possible. Even with semi-continuity at a point one may have invariance for $I$ without having it for $R$. Also invariance for $I$ alone does not suffice to derive continuity of $R$ from semi-continuity at some point.

The continuity of $R$ has been used in a twofold way.

First, the closedness of indifference sets $I_{x}, x \in \mathbb{R}^{\prime}$ was caused by continuity. Secondly, the ordering of the closed indifference manifolds in such a way that "above" each indifference set are only better elements and "below" are only worse ones is also due to continuity.

Without continuity of $R$ we are left with co-sets $I_{x}, x \in \mathbb{R}^{t}$, of a subgroup $I_{0}$ of $\mathbb{R}^{\ell}$ which, however, need not be closed. Also the indifference manifolds (even when closed) could be ordered in quite an arbitrary way.

This is possible since the continuity of $R$ which is equivalent to the continuity of its representation $u: \mathbb{R}^{\prime} \rightarrow \mathbb{R}$ amounts to the simultaneous continuity of both of 
the two maps proj and $\tilde{u}$ into which $u$ can be decomposed as:

$$
\mathbb{R}^{\ell} \stackrel{\text { proj }}{\longrightarrow} \mathbb{R}^{\ell} / I \stackrel{\tilde{u}}{\longrightarrow} \mathbb{R}: x \stackrel{\text { proj }}{\longrightarrow} I_{x} \stackrel{\tilde{u}}{\longrightarrow} u(x) .
$$

Here $\mathbb{R}^{\ell} / I$ is endowed with the identification topology.

Exploiting the isomorphism between the topological groups $\left(\mathbb{R}^{\ell},+\right)$ and $\left(\mathbb{R}_{++}^{\ell}, \cdot\right)$ described by the coordinatewise exponential map, one can restate the above results as statements about preferences which are invariant under stretching of the axes of the space.

This invariance called budget-invariance has been used to characterize CobbDouglas representable preferences by continuity, monotonicity and price-invariance. The present Corollary allows to extend that result (cf. Trockel (1989)) to nonmonotonic preferences.

\section{References}

Blackwell, D., Girshick, M.A.: Theory of games and statistical decisions. New York: John Wiley \& Sons, 1954

d'Aspremont, C.: Axioms for social welfare orderings. In: Hurwicz, L., Schmeidler, D., Sonnenschein, H. (eds.) Social goals and social organization. Cambridge: Cambridge University Press, 1985

d'Aspremont, C., Gevers, L.: Equity and the informational basis of collective choice. Rev. Econ. Stud. 44, 199-209 (1977)

Gevers, L.: On interpersonal comparability and social welfare orderings. Econometrica 47,75-89(1979)

Herstein, I.N., Milnor, J.: An axiomatic approach to measurable utility. Econometrica, 21, 291-297(1953)

Maskin, E.: A theorem on utilitarianism. Rev. Econ. Stud. 45, 93-96 (1978)

Roberts, K.W.S.: Interpersonal comparability and social choice theory. Rev. Econ. Stud. 47, 421-439 (1980)

Trockel, W.: Classification of budget-invariant monotonic preferences. Econ. Lett. 30, 7-10 (1989) 